eCommons@AKU

April 2018

\title{
Diagnostic accuracy of pelvic MRI for determination of the cervical involvement in endometrial cancer.
}

Imrana Masroor

Aga Khan University, imrana.masroor@aku.edu

Sadia Rashid

Aga Khan University

Shaista Afzal Saeed

Aga Khan University, shaista.afzal@aku.edu

Saira Naz

Aga Khan University, Saira.naz@aku.edu

Muhammad Azeemuddin

Aga Khan University, muhammad.azeemuddin@aku.edu

Follow this and additional works at: https://ecommons.aku.edu/pakistan_fhs_mc_radiol

Part of the Radiology Commons

\section{Recommended Citation}

Masroor, I., Rashid, S., Saeed, S., Naz, S., Azeemuddin, M. (2018). Diagnostic accuracy of pelvic MRI for determination of the cervical involvement in endometrial cancer.. Journal of the College of Physicians and Surgeons--Pakistan, 28(4), 262-265.

Available at: https://ecommons.aku.edu/pakistan_fhs_mc_radiol/114 


\title{
Diagnostic Accuracy of Pelvic MRI for Determination of the Cervical Involvement in Endometrial Cancer
}

\author{
Imrana Masroor, Sadia Rashid, Shaista Afzal, Saira Naz Sufian and Muhammad Azeemuddin
}

\begin{abstract}
Objective: To determine the diagnostic accuracy of pelvic MRI for assessment of the cervical involvement in endometrial cancer.

Study Design: Cross-sectional analytical study.

Place and Duration of Study: Radiology Department of the Aga Khan University Hospital, Karachi from January 2014 to December 2015.

Methodology: Patients with biopsy-proven endometrial cancer were included, who had both their MRI and histopathological diagnosis performed at our institution. Those patients treated with chemo/radiotherapy or had incomplete medical records, were excluded. The extent of cervical involvement by endometrial carcinoma was seen on T2WI images, and findings were correlated after surgery taking histopathology as the gold standard. The sensitivity, specificity, positive predictive value, negative predictive value and diagnostic accuracy were calculated.

Results: The mean age of the 56 patients was $60.87 \pm 8.80$ years (range 37-84 years). The most common clinical indication was post-menopausal bleeding $(n=37,66.1 \%)$. The most common histological subtype was endometrioid adenocarcinoma $(n=50,89.3 \%)$. The sensitivity, specificity, diagnostic accuracy, positive and negative predictive values of MRI in the detection of cervical invasion were $92.85 \%, 88.09 \%, 89.28 \%, 72.22 \%$ and $97.36 \%$, respectively.

Conclusion: MRI is a highly sensitive and specific imaging modality for detection of cervical invasion in endometrial carcinoma.
\end{abstract}

Key Words: Endometrial carcinoma. Cervical involvement. Magnetic resonance imaging. Diagnostic accuracy.

\section{INTRODUCTION}

The most common gynecological malignancy and the fourth most common cancer of female in the United States is endometrial carcinoma. ${ }^{1}$ Internationally, it makes up $6 \%$ of all cancers in females. ${ }^{2}$ It has an incidence of 5.9 per 100,000 women in developing countries, and the second most common gynecological malignancy. Endometrial cancer is diagnosed mainly during the 6 th and 7 th decades. ${ }^{3}$ The 10 -year survival rate is $75 \%$, because $75-80 \%$ of women present with symptoms of postmenopausal bleeding at an early stage. 4

The risk factors associated with the development of endometrial cancer include obesity, nulliparity unopposed estrogen intake, Stein-Leventhal syndrome, diabetes mellitus, Lynch syndrome, and tamoxifen therapy. 5 Diagnosis, however, is done with either hysteroscopicguided endometrial biopsy or dilation and curettage.

Histologically, there are two subtypes of endometrial cancer. ${ }^{6}$ The most frequent is endometrioid adenocarcinoma (Type I) which is $90 \%$ of all endometrial

Department of Radiology, The Aga Khan University Hospital,

Karachi.

Correspondence: Dr. Muhammad Azeemuddin, Associate

Professor, Department of Radiology, The Aga Khan University

Hospital, Stadium Road, Karachi.

E-mail: muhammad.azeemuddin@aku.edu

Received: October 17, 2017; Accepted: February 02, 2018. tumors. ${ }^{3}$ In Pakistan, adenocarcinoma is the most common histological type accounting for $85.8 \% .7,8$ Endometrial cancer frequently occurs in a background of endometrial hyperplasia, which is common in the beginning of menopause, are usually low grade, and show a good prognosis. ${ }^{9}$ Type II endometrial cancers include all other histological subtypes namely clear-cell, serous papillary and carcinosarcomas. Type II cancers often occur in elderly females, show no relationship with increased levels of estrogen or atypical hyperplasia, have a poorer prognosis, and spread like ovarian cancer. ${ }^{10}$

In 1988, surgicopathologic International Federation of Gynecology and Obstetrics (FIGO) devised staging for endometrial cancer. ${ }^{11}$ In 2009, few specific changes were made in staging. ${ }^{12,13}$ In the new staging system, stage I was further divided into two substages: la (showing equal/less than $50 \%$ myometrial invasion), and $\mathrm{Ib}$ (showing equal/greater than 50\% myometrial invasion), in contrast with the older system, which had three sub-stages; la (confined to endometrium), lb (showing less than $50 \%$ myometrial invasion), and Ic (showing equal/greater than $50 \%$ myometrial invasion). There are no sub-stages for stage II, and only cervical stromal involvement (stage Illb, old system) is considered and endo-cervical glandular invasion (stage Ila, old system) is now diagnosed as stage I endometrial cancer. ${ }^{11}$

Accurate staging of endometrial cancer is crucial at the time of diagnosis to successfully manage patients and 
thus increase survival rate. ${ }^{11} \mathrm{~A}$ study conducted by Taufiq et al. reported a potential role of MRI for determining myometrial invasion. ${ }^{14}$ The data about the role of Magnetic Resonance Imaging (MRI) in staging of endometrial cancer, particularly in detection of cervical invasion in our country, is limited.

The aim of this study was to determine the sensitivity, specificity, positive predictive value (PPV), negative predictive value (NPV) and accuracy of MRI in the detection of cervical involvement by endometrial carcinoma.

\section{METHODOLOGY}

It was a cross-sectional analytical study conducted at the Department of Radiology, The Aga Khan University Hospital (AKUH), Karachi, Pakistan from January 2014 to December 2015. Exemption was taken for this retrospective study from Ethical Review Committee of the Hospital. The sample size was calculated using the Lin Naing sample size calculator. The sensitivity and specificity of MRI T2 weighted images (T2WI) for determination of cervical invasion has been calculated to be $100 \%$ and $95 \%$, respectively; the reported prevalence of endometrial cancer is $6 \%$. Therefore with a confidence interval of $95 \%$, and a $6 \%$ error margin, the calculated sample size was 56 patients. Purposive nonprobability sampling was applied. The inclusion criteria were all patients (all ages) with histology proven endometrial carcinoma on biopsy, and had their MRI pelvis for staging before hysterectomy, who then underwent surgery and histopathology at our hospital. All patients treated with radiotherapy/chemotherapy or patients with incomplete medical records were excluded. The final histological diagnoses, i.e. invasion of cervix and final staging of patients, was recorded.

Imaging protocol included all routine sequences comprising coronal T1W, axial, sagittal, coronal T2W, axial T1W fat suppressed, and sagittal T1W postcontrast with fat suppression images. T2W imaging was done by the protocol set in our department for female pelvis, with the following parameters: TR/TE 3900/76; field of view $38 \mathrm{~cm}$; slice thickness $4 \mathrm{~mm}$; gap 0 ; number of excitations 4; matrix 192x192; 3 acquisitions with 8 slices per acquisitions; and phase encoding direction, anterior to posterior. The time needed to obtain the T2W images was set to 4 minutes.

Scan reading was performed by two independent radiologists with greater than 5 years of experience in women imaging. MRI results were compared with final histopathology setting letter as gold standard. True positive was taken if abnormal signal of endometrium, i.e. hyperintense to myometrium and hypointense to normal endometrium, was seen extending into cervix on $\mathrm{T} 2 \mathrm{WI}$, and cervical invasion was reported on histopathology. True negative was taken if abnormal signal of endometrium, i.e hyperintense to myometrium and hypointense to endometrium, was not extending into cervix and invasion of cervix not seen on histopathology.

Statistical analysis was performed on SPSS (version 21.0). Mean and standard deviation were calculated for all continuous variables and frequencies with percentages were calculated for categorical ones.

Sensitivity, specificity, Positive Predictive Value (PPV), Negative Predictive Value (NPV) and accuracy were determined. Positive cases comprised true positives and false negatives; and negative cases comprised true negatives and false positives.

\section{RESULTS}

Fifty-six patients had a mean age of $60.87 \pm 8.80$ years (range 37-84 years). The most common clinical indication was post-menopausal bleeding $(n=37,66.1 \%)$ followed by increased endometrial thickness on ultrasound $(n=11,19.6 \%)$ and irregular vaginal bleeding in premenopausal patients $(n=4,7.1 \%)$. The most common histological subtype was the endometrioid adenocarcinoma detected in $89.3 \%(n=50)$ of the patients.

In MRI on T2W images, endometrial carcinoma appeared heterogeneous in 23 patients (Figure 1, 41.1\%); hypointense in 24 patients (42.9\%); and hyperintense in nine patients $(16.1 \%)$. On diffusion weighted images (DWI), 52 patients $(92.9 \%)$ showed diffusion restriction and four patients $(7.1 \%)$ were negative for diffusion restriction. On T1W fat sat post contrast images, 26 cases $(46.4 \%)$ did not show enhancement, 25 cases (44.6\%) showed mild enhancement, and five cases $(8.9 \%)$ showed heterogeneous enhancement. On T2W images, cervical involvement was detected in 18 patients (32.1\%); while in 38 patients (67.9\%), cervical involvement was not detected.

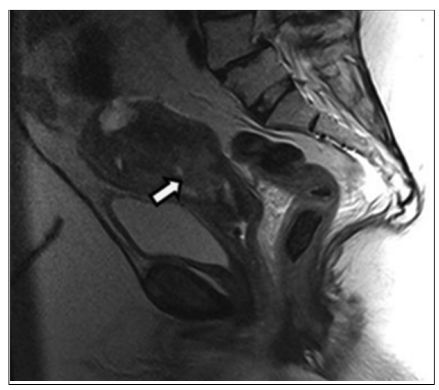

Figure 1: T2 weighted image demonstrating anteverted uterus showing neoplastic mass (arrow) involving the endometrium and the cervix.

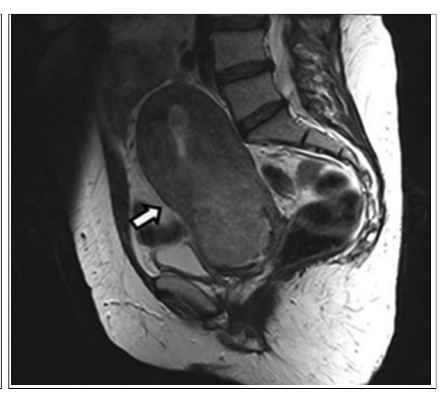

Figure 2: T2 weighted image demonstrating anteverted uterus showing neoplastic mass (arrow) involving the endometrium extending up to the cervix.
On histopathology report, cervical involvement was detected in 15 patients $(26.8 \%)$; while in 41 patients $(73.2 \%)$, cervical involvement was not detected. A total of 13 out of 18 patients $(72.2 \%)$, who showed cervical invasion on the T2W MRI (Figure 2), turned out to be positive on histopathology (true positive) and 5 out of 18 
Table I: Accuracy of pelvic MRI for assessment of the cervical involvement in endometrial cancer.

\begin{tabular}{|c|c|c|c|}
\hline \multirow{2}{*}{ T2W MRI cervical invasion } & \multicolumn{3}{|c|}{ Histopathology cervical invasion } \\
\hline & Positive & Negative & Tota \\
\hline Positive & 13 & 05 & 18 \\
\hline Negative & 01 & 37 & 38 \\
\hline Total & 14 & 42 & 56 \\
\hline
\end{tabular}

patients $(27.7 \%)$ who showed cervical invasion on $\mathrm{T} 2 \mathrm{~W}$ MRI, were negative on histopathology (false positive).

Out of 38 patients, 37 (97.3\%) did not show cervical invasion on MRI, were negative for cervical invasion on histopathology (true negative); while one out of these 38 patients $(2.63 \%)$ who were negative on MRI for cervical invasion came out to be positive for cervical invasion on histopathology (false negative).

In evaluation of cervical infiltration, the sensitivity, specificity, positive, negative predictive and diagnostic accuracy, values of T2W MRI with $95 \%$ confidence interval were $92.85 \%, 88.09 \%, 72.22 \%, 97.36 \%$ and $89.28 \%$, respectively (Table I).

\section{DISCUSSION}

There are various imaging modalities such as ultrasound and computed tomography (CT) scan available for the assessment of endometrial carcinoma but MRI gives far more information with regard to its extent which helps in treatment planning and determining prognosis.

Transvaginal ultrasound (TVUS) is a rapid, low-cost, non-ionizing modality often used as a first imaging in initial workup of women with complaint of postmenopausal bleeding. ${ }^{3}$ Endometrial carcinoma is typically visualised as thickening of the endometrial lining, and TVUS is used to measure this thickened endometrial lining in the anterio-posterior diameter, to reach a diagnosis. The sensitivity and specificity of TVUS for detecting endometrial carcinoma approach $96 \%$ and $61 \%$, respectively, when endometrial thickness of $5 \mathrm{~mm}$, in postmenopausal women, is used to define abnormal endometrial thickening. ${ }^{13}$ But it is hard to demarcate the tumor margins on TVUS, particularly when the tumor is diffusely infiltrating the myometrium. ${ }^{15}$ Drawbacks of TVUS are that it is highly operatordependent, and has limited field of view. It may overestimate myometrial invasion when the tumor is large, or patient has adenomyosis, and lymphovascular invasion. Additionally, there is inadequate information about the role of TVUS in delineating cervical invasion, parametrial extension, or lymph node involvement. 16

On contrast enhanced Computed Tomography, endometrial carcinoma is seen as a hypodence and less enhancing area within the endometrial cavity. This appearance is not specific enough, thus differential diagnoses such as sub-mucosal leiomyomas, endometrial polyps etc. cannot be fully excluded. ${ }^{3}$ CT shows less sensitivity and specificity in correctly determining myometrial involvement and cervical invasion as opposed to MRI. Therefore, it appears to have a limited role in local staging due to its suboptimal soft tissue delineation. The sensitivity and specificity of CT in determining myometrial involvement ranges from $40 \%$ to $83 \%$ and $42 \%$ to $75 \%$, respectively. 17 Recent studies done to assess preoperatively, the myometrial and cervical extension of endometrial carcinoma with multidetector CT showed better diagnostic accuracy of $95 \%$ and $81 \%$, respectively. 18 At present, CT is used generally in the assessment of advanced stage disease, i.e. for evaluating patients with endometrial carcinoma by determining nodal and distant metastases.

MRI achieves the greatest accuracy, for pre-treatment local staging of endometrial cancer, mainly due to its superb soft tissue delineation. On MRI, endometrial carcinoma is usually detected as a hypo-to-isointense mass on T1WI, showing intermediate signal intensity less than the normal endometrium on T2WI. ${ }^{3}$ On postcontrast dynamic imaging, endometrial carcinoma enhances a lesser amount than the myometrium. On the whole, staging accuracy of MRI is reported to be 83$92 \% .{ }^{19,20}$

The usual signal of normal cervical stroma on T2WI is low, this becomes distinct to the high-intermediate signal intensity of tumor infiltration. ${ }^{21}$ The sensitivity, specificity, and diagnostic accuracy of MR in determination of cervical infiltration have been reported to be $100 \%, 87 \%$, and $90 \%$, respectively and on $\mathrm{T} 2 \mathrm{WI} 100 \%, 95 \%$, and $96 \%$, respectively. 22

In this study, most of the patients were post-menopausal and presented with post-menopausal bleeding. Endometrial carcinoma was detected on T2WI, DWI as well as on T1W fat sat post contrast images but cervical invasion was best detected on $\mathrm{T} 2 \mathrm{~W}$ images with diagnostic accuracy approaching $89.28 \%$.

In a study conducted by Zamani et al., the sensitivity and specificity in cervical stromal infiltration was higher in distinction to mucosal infiltration, $54.54 \%$ and $100 \%$ vs. $71.4 \%$ and $97.5 \%$, respectively. ${ }^{11}$

In another study conducted by Seki et al., the overall accuracy of MRI in predicting cervical infiltration was determined to be $90-92 \%$, with sensitivity of $75-80 \%$ and specificity of $94-96 \% .23$

This study shows the sensitivity and specificity for cervical stromal involvement as $92.85 \%$ and $88.09 \%$, respectively. The sensitivity of this study was higher than studies conducted by Zamani et al. and Seki et al. probably because of the larger sample size.

MRI's greater soft tissue delineation allows it to be a superior than other cross-sectional imaging modalities in determining adnexal metastases, invasion of vagina, 
urinary bladder, and rectum. It is thought that with the new 2009 FIGO staging system, an improved accuracy in MRI staging is obtained. 24,25

The main limitation of this study was the inter-observer agreement between the two consultants, which was not taken into account. Furthermore, the patients who underwent curettage before MRI were not excluded from this study; thus the imaging performed after cervical curettage may result in abnormal signal intensity, replacing the normal hypointense signal intensity of cervix.

\section{CONCLUSION}

At present, MRI is an accurate, sensitive and specific modality for cervical invasion of endometrial carcinoma.

\section{REFERENCES}

1. National Cancer Institute. Cancer stat fact sheets: sorpus and uterus, NOS. Bethesda, Md: National Cancer Institute, 2010

2. American Cancer Society. Cancer facts and figures. Atlanta, GA: American Cancer Society; 1997

3. Faria SC, Sagebiel T, Balachandran A, Devine C, Lal C, Bhosale PR. Imaging in endometrial carcinoma. Indian $\mathrm{J}$ Radiol Imaging 2015; 25:137.

4. Cancer Research UK. Cancerstats. Available via http://info. Cancerresearchuk.org/cancerstats/.

5. Arora V, Quinn MA. Endometrial cancer. Best Pract Res Clin Obstet Gynaecol 2012; 26:311-24.

6. Wright JD, BarrenaMedel NI, Sehouli J, Fujiwara K, Herzog TJ. Contemporary management of endometrial cancer. Lancet 2012; 379:1352-60.

7. Farhat N. Pattern of gynecological malignancies in tertiary hospital. J postgrad Med Inst 2002; 16:215-20.

8. Abdul RS, Sher MS, Rafia B, Abdul WS. Gynecological cancer: A histological experience at Chandka Medical College and Hospital Larkana. Med Channal 1999; 5:15-9.

9. Tirumani SH, Shanbhogue AK, Prasad SR. Current concepts in the diagnosis and management of endometrial and cervical carcinomas. RadiolClin North Am 2013; 51:1087-110.

10. Creasman W. Revised FIGO staging for carcinoma of the endometrium. Int J Gynaecol Obstet 2009; 105:109.

11. Zamani F, Goodarzi S, Hallaji F, Zamiri A, Deilami T, Malek M, et al. Diagnostic value of pelvic MRI for assessment of the depth of myometrial invasion and cervical involvement in endometrial cancer: Comparison of new versus old FIGO staging. Iran J Radiol 2012; 9:202-8.
12. Creasman WT, Odicino F, Maisonneuve $P$, Quinn MA, Beller U, Benedet JL, et al. Carcinoma of the corpus uteri. FIGO 26th annual report on the results of treatment in gynecological cancer. Int J Gynaecol Obstet 2006; 95(Suppl 1):S105-43.

13. Taufiq M, Masroor I, Hussain Z. Daignostic accuracy of diffusion weighted MR imaging in detection of myometrial invasion in endometrial carcinoma. J Coll Physicians Surg Pak 2016; 1:13-7.

14. Smith-Bindman R, Kerlikowske K, Feldstein VA, Subak L, Scheidler J, Segal M, et al. Endovaginal ultrasound to exclude endometrial cancer and other endometrial abnormalities. JAMA 1998; 280:1510-7.

15. Ascher SM, Reinhold C. Imaging of cancer of the endometrium. Radiol Clin North Am 2002; 40:563-76.

16. Barwick TD, Rockall AG, Barton DP, Sohaib SA. Imaging of endometrial adenocarcinoma. Clin Radiol 2006; 61:545-55.

17. Hardesty LA, Sumkin JH, Hakim C, Johns C, Nath M. The ability of helical CT to preoperatively stage endometrial carcinoma. AJR Am J Roentgenol 2001; 176:603-6.

18. Tsili AC, Tsampoulas C, Dalkalitsis N, Stefanou D, Paraskevaidis E, Efremidis SC. Local staging of endometrial carcinoma: Role of multidetector CT. Eur Radiol 2008; 18: 1043-8.

19. Akin O, Mironov S, Pandit-Taskar N, Hann LE. Imaging of uterine cancer. Radiol Clin North Am 2007; 45:167-82.

20. Hirano Y, Kubo K, Hirai Y, Okada S, Yamada K, Sawano S, et al. Preliminary experience with gadolinium-enhanced dynamic MR imaging for uterine neoplasms. Radiographics 1992; 12: 243-56.

21. Hricak H, Rubinstein LV, Gherman GM, Karstaedt N. MRI evaluation of endometrial carcinoma: Results of an $\mathrm{NCl}$ cooperative study. Radiology 1991; 179:829-32.

22. Lien HH, Blomlie V, Trope C, Kaern J, Abeler VM. Cancer of the endometrium: Value of MR imaging in determining depth of invasion into the myometrium. AJR Am J Roentgenol 1991; 157:1221-3

23. Seki H, Azumi R, Kimura M, Sakai K. Stromal invasion by carcinoma of the cervix: assessment with dynamic MR imaging. AJR Am J Roentgenol 1997; 168:1579-85.

24. Murakami T, Kurachi H, Nakamura H, Tsuda K, Miyake A, Tomoda K, et al. Cervical invasion of endometrial carcinoma evaluation by parasagittal MR imaging. Acta Radiol 1995; 36:248-53.

25. Ballester M, Koskas M, Coutant C, Chereau E, Seror J, Rouzier R, et al. Does the use of the 2009 FIGO classification of endometrial cancer impact on indications of the sentinel node biopsy? BMC Cancer 2010; 10:465. 